Pacific Journal of Mathematics

A NOTE ON PRINCIPAL FUNCTIONS AND MULTIPLY-VALENT
CANONICAL MATINGS 


\title{
A NOTE ON PRINCIPAL FUNCTIONS AND MULTIPLY- VALENT CANONICAL MAPPINGS
}

\author{
PAUL A. Nickel
}

\begin{abstract}
L. Sario has constructed principal analytic functions on planar bordered Riemann surfaces by applying the method of linear operators to certain sets of singularity functions. Weakly $\lambda$-valent principal functions result from a similar construction, starting with singularity functions having flux equal to integral multiples of $2 \pi$. In fact, such $\lambda$-valent maps are characterized as integral powers of principal analytic functions already mentioned.
\end{abstract}

L. Sario has used linear operators to establish the existence of certain canonical mappings of planar bordered Riemann surfaces $\bar{W}$ onto slit disks [4]. These mappings $F_{0}(z)$ and $F_{1}(z)$, called principal analytic functions, are formed from principal harmonic functions, themselves constructed by applying the linear operator method of [5] to systems of singularity functions defined near certain point sets of $\bar{W}$. In particular, near $\gamma$, the border of $\bar{W}$, the singularity function $s_{\gamma}(z)$, which is constant on $\gamma$ with flux $2 \pi$ there, is chosen, while near $\zeta$, a point of the surface $W=\bar{W}-\gamma$, the singularity function $s_{\zeta}(z)=$ $\log |z-\zeta|$ is selected. By exhausting the planar bordered surface $\bar{W}$, one constructs the mappings $F_{0}(z)$ and $F_{1}(z)$ of $\bar{W}$ onto a plane disk, with radial or circular slits, possibly degenerate. It is easily established that, for $i=0,1, \Delta_{\gamma}\left(\arg F_{i}(z)\right)$ is $2 \pi$, the flux on $\gamma$ of the singularity function $s_{\gamma}(z)$, and that each $F_{i}(z)$ has a first order zero at $z=\zeta$. These conditions are easily seen to be a consequence of the selection of the singularity functions $s_{\gamma}(z)$ and $s_{\zeta}(z)$.

In this note, we investigate the nature of "canonical" maps $F_{0}^{\lambda}(z)$ and $F_{1}^{\lambda}(z)$ which result from starting with singularity functions $s_{\gamma}^{\lambda}(z)$ near $\gamma$ and $s_{\zeta}^{\lambda}(z)$ near $\zeta$. Here, $s_{\gamma}^{\lambda}(z)$ is constant for $z \in \gamma$ with flux $\int_{\gamma} d s_{\gamma}^{\lambda *}=2 \pi \lambda$ while $s_{\zeta}^{\lambda}(z)=\lambda \log |z-\zeta|$. If an approximation process similar to that of [4] is applied, canonical maps $F_{0}^{\lambda}(z)$ and $F_{1}^{\lambda}(z)$ result. Because $\Delta_{\gamma} \arg F_{i}(z)=\int_{\gamma} d s_{\gamma}^{\lambda *}=2 \pi \lambda$, it follows that the mappings $F_{0}^{\lambda}(z)$ and $F_{1}^{\lambda}(z)$ are $\lambda$-valent, at least near $\gamma$. Also, at the point $\zeta$ of $W$, these mappings have a $\lambda$-th order zero, and hence are $\lambda$-valent near $\zeta$ as well. It is then reasonable to ask whether the functions $F_{0}^{\lambda}(z)$ and $F_{1}^{\lambda}(z)$, with radial and circular slit behavior near the ideal boundary, are globally $\lambda$-valent in some sense.

For a bordered Riemann surface $\bar{V}$ with two border components $\delta$ and $\gamma$, constructions similar to those of [3], starting with singularity 
functions $s_{\delta}^{\lambda}(z)$ and $s_{\gamma}^{\lambda}(z)$, lead to similar questions concerning the nature of $\lambda$-valent mappings of $\bar{V}$ onto a slit annulus. The purpose of this note then, is to determine the geometric nature of multiplyvalent canonical mappings based on the constructions already outlined. In terms of principal functions already known, we shall be able to establish a classification of such mappings based on the concept of weak $\lambda$-valence.

2. The $\lambda$-th principal analytic functions. We consider first an exhausting set of bordered surfaces $\left\{\bar{W}_{n}\right\}$, each of which has $\gamma$ as one of its border components, and has remaining border components denoted $\beta_{1}, \cdots, \beta_{k(n)}$. On every $\bar{W}_{n}$, we construct $\lambda$-th principal analytic functions $F_{0 n}^{\lambda}(z)$ and $F_{1 n}^{\lambda}(z)$ such that (i) $\left|F_{i n}^{\lambda}(z)\right|=$ constant = $r_{\gamma}\left(F_{i n}^{\lambda}\right)$ for $z \in \gamma$ with $\Delta_{\gamma}\left(\arg F_{i n}^{\lambda}(z)\right)=2 \pi \lambda$, (ii) $F_{i n}^{\lambda}(z)$ has a $\lambda$-th order zero at $z=\zeta$, and (iii) $F_{0 n}^{\lambda}(z)\left(F_{1 n}^{\lambda}(z)\right)$ maps each of $\bar{W}_{n}^{\prime}$ s remaining border components $\beta_{i}$ onto a radial (circular) slit. Such mappings are constructed by selecting singularity functions $s_{\gamma}^{\lambda}(z)$ and $s_{\zeta}^{\lambda}(z)$ already defined in $\S 1$, and selecting the singularity functions $s_{\beta_{i}}^{\lambda}(z)$ near $\beta_{i}$ in the manner of [4]. For $i=0,1$, the functions $F_{i n}^{\lambda}(z)$ result from an application of the linear operator method [5], and these are normalized by the condition $\lim _{z \rightarrow \zeta} F_{i n}^{\lambda}(z) /(z-\zeta)^{\lambda}=1$. The families $\left\{F_{0 n}^{\lambda}(z)\right\}$ and $\left\{F_{1 n}^{\lambda}(z)\right\}$ are normal, and the resulting limits $F_{0}^{\lambda}(z)$ and $F_{1}^{\lambda}(z)$ are called $\lambda$-th principal analytic functions on $\bar{W}$. It now seems reasonable to expect that these mappings are weakly $\lambda$-valent in the following sense.

Definition. The mapping $F(z)$ is called weakly $\lambda$-valent if, for each $w \in F(\bar{W})$, the set $F^{-1}(w)$ consists of at most $\lambda$ points $z \in \bar{W}$, and for some $w \in F(\bar{W})$, the set $F^{-1}(w)$ consists of exactly $\lambda$ points. A weakly $\lambda$-valent mapping $F(z)$ of $\bar{W}$ into the point set $S$ is called a radial (circular) slit mapping of $\bar{W}$ into $S$ if each component of the set $\left\{w \in S ; F^{-1}(w)\right.$ contains at most $\lambda-1$ points $\left.z \in \bar{W}\right\}$ is a radial (circular) slit or point.

3. Properties of $\lambda$-th principal analytic functions. The following are some properties of the maps $F_{0}^{\lambda}(z)$ and $F_{1}^{\lambda}(z)$ which will prove useful.

(i) For $i=0,1$, the function $F_{i}^{\lambda}(z)$ has no zero on the surface $W-\zeta$.

(ii) If $\Delta(\zeta)$ is a parametric disc with boundary $\delta$ whose orientation is induced by $\Delta(\zeta)$, then for $i=0,1, \int_{\delta} d\left(\arg F_{i}^{\lambda}(z)\right)=2 \pi \lambda$.

(iii) If $\sigma$ is a cycle contained in $W-\zeta$, then for $i=0,1, \lambda$ divides the integer $(1 / 2 \pi) \int_{\sigma} d\left(\arg F_{i}^{\lambda}(z)\right)$. 
Proof of (i). If $Z_{n}$ is the number of zeros of $F_{i}^{\lambda}(z)$ in $\bar{W}_{n}$, with border $\gamma+\bar{\beta}_{n}$, we apply the argument principle and find:

$$
\begin{aligned}
Z_{n} & =\frac{1}{2 \pi i} \int_{\gamma+\bar{\beta}_{n}} \frac{d F_{i}^{\lambda}(z)}{d z} / F_{i}^{\lambda}(z) d z=\lim _{m} \frac{1}{2 \pi i} \int_{\gamma+\bar{\beta}_{n}} \frac{d F_{i m}^{\lambda}(z)}{d z} / F_{i m}^{\lambda}(z) d z \\
& =\lim _{m} \frac{1}{2 \pi} \int_{\gamma+\bar{\beta}_{n}} d\left(\arg F_{i m}^{\lambda}(z)\right)=\lambda .
\end{aligned}
$$

Hence $F_{i}^{\lambda}(z)$ is never zero on the surface $W-\zeta$.

Proof of (ii). In the parametric disk $\Delta(\zeta)$, we may write $F_{i}^{\lambda}(z)=$ $(z-\zeta)^{\lambda} f_{i}(z)$, where $f_{i}(z)$ is never zero in $\overline{\Delta(\zeta)}$. Hence it follows that $\int_{\delta} d\left(\arg F_{i}(z)\right)=2 \pi \lambda$.

Proof of (iii). We let $\sigma$ be an arbitrary cycle of $W-\zeta$, and choose $n$ large enough so that $\sigma \subset \bar{W}_{n}$. If a parametric disk $\Delta(\zeta)$ is removed from $\bar{W}_{n}$, the bordered $\bar{W}_{n}-\Delta(\zeta)$ results. Hence there are integers $a, b$, and $c_{i}$ such that $\sigma$ is homologous (in $\bar{W}_{n}-\Delta(\zeta)$ ) to $a \gamma+b \delta+\sum_{1}^{k(n)} c_{j} \beta_{j}$; and $\int_{\sigma} d\left(\arg F_{i}^{\lambda}(z)\right)$ may be written as

$$
\begin{aligned}
\int_{\sigma} d\left(\arg F_{i}^{\lambda}(z)\right)= & a \int_{\gamma} d\left(\arg F_{i}^{\lambda}(z)\right)+b \int_{\delta} d\left(\arg F_{i}^{\lambda}(z)\right) \\
& +\sum_{1}^{k(n)} c_{j} \int_{\beta_{j}} d\left(\arg F_{i}^{\lambda}(z)\right) \\
= & 2 \pi \lambda a-2 \pi \lambda b+\lim _{m} \sum_{1}^{k(n)} c_{j} \int_{\beta_{j}} d\left(\arg F_{i m}^{\lambda}(z)\right) \\
= & 2 \pi \lambda a-2 \pi \lambda b .
\end{aligned}
$$

Thus it follows that $\lambda$ divides $(1 / 2 \pi) \int_{\sigma} d\left(\arg F_{i}^{\lambda}(z)\right)$.

The following theorem, characterizing the nature of $F_{0}^{\lambda}(z)$ and $F_{1}^{\lambda}(z)$, is our main result.

THEOREM 1. The $\lambda$-th principal analytic function $F_{0}^{\lambda}(z)\left(F_{1}^{\lambda}(z)\right)$ is the $\lambda$-th power of the principal analytic function $F_{0}(z)\left(F_{1}(z)\right)$ of [4].

Proof. We consider only the mapping $F_{0}^{\lambda}(z)$ because the arguments for $F_{1}^{\lambda}(z)$ are entirely analogous. According to property (iii) of this section, $\lambda$ divides $(1 / 2 \pi) \int_{\sigma} d\left(\arg F_{0}^{\lambda}(z)\right)$ for each cycle $\sigma$ in $W-\zeta$. Hence it follows from the theorem of the appendix that $F_{0}^{\lambda}(z)$ has an analytic $\lambda$-th root, say $G(z)$, in $W-\zeta$. But $\zeta$ is a removable singularity for $G(z)$, and we call $G(z)$, with $G(\zeta)=0$, an analytic $\lambda$-th 
root of $F_{0}^{\lambda}(z)$ in $W$, and in fact in $\bar{W}$.

In the neighborhood $\Delta$ of $\zeta$, we have $F_{0}^{\lambda}(z)=(G(z))^{\lambda}=(z-\zeta)^{\lambda} H(z)$, where $H(\zeta)$ has been normalized to 1 . If we let $\widetilde{H}(z)$ be some analytic $\lambda$-th root of $H(z)$, then the set of functions $\{\widetilde{H}(z) \exp 2 \pi i k / \lambda ; k=$ $0,1, \cdots, \lambda-1\}$, according to the corollary in the appendix, represents all $\lambda$-th roots. But $(\tilde{H}(\zeta))^{\lambda}=1$, hence one of the quantities $\widetilde{H}(\zeta) \exp 2 \pi i k / \lambda$ is 1 . We assume that this occurs for $k=0$, that is, $\tilde{H}(\zeta)=1$, and we take $G(z)$, with $(G(z))^{\lambda}=F_{0}^{\lambda}(z)$, as that branch for which $\tilde{H}(\zeta)=1$. In particular, in $\Delta(\zeta), G(z)=(z-\zeta) \widetilde{H}(z)$, and $G^{\prime}(\zeta)=1$.

We claim now that the functional $\Phi(G)=2 \pi \log r(G)-A(G)$ of [4] has the value $\Phi\left(F_{0}\right)$, where $F_{0}$ is the univalent principal radial slit mapping of [4]. To see this, we need only compute the deviation $\Phi\left(F_{0}\right)-\Phi(G)$, and according to Theorem 3 of [4], this is only $D_{\bar{W}}\left(\log \left|G(z) / F_{0}(z)\right|\right)$. But $\log \left|G(z) / F_{0}(z)\right|$ has a removable singularity at $\zeta$, hence we write

$$
\begin{aligned}
\lambda^{2} D_{\bar{W}}\left(\log \left|\frac{G(z)}{F_{0}(z)}\right|\right) & =D_{\bar{W}}\left(\log \frac{\left|F_{0}^{\lambda}(z)\right|}{\left|F_{0}(z)\right|^{\lambda}}\right) \\
& =\int_{\gamma} \log \frac{\left|F_{0}^{\lambda}(z)\right|}{\left|F_{0}(z)\right|^{\lambda}} d\left(\arg \frac{F_{0}^{\lambda}(z)}{\left(F_{0}(z)\right)^{\lambda}}\right) \\
& +\int_{\beta} \frac{\left|F_{0}^{\lambda}(z)\right|}{\left|F_{0}(z)\right|^{\lambda}} d\left(\arg \frac{F_{0}^{\lambda}(z)}{\left(F_{0}(z)\right)^{\lambda}}\right) \\
= & \log \frac{r_{\gamma}\left(F_{0}^{\lambda}\right)}{\left(r_{\gamma}\left(F_{0}\right)\right)^{\lambda}}(2 \pi \lambda-2 \pi \lambda)=0 .
\end{aligned}
$$

It now follows from reasoning similar to the proof of Theorem 3 in [4] that $G(z)$, and only $G(z)$, maximizes $\Phi$ among analytic functions $F$ satisfying ( i) $F(z)=$ const for $z \in \gamma$ and $\int_{\gamma} d(\arg F(z))=2 \pi$. (ii) $F(\zeta)=0$ and $F^{\prime}(\zeta)=1$, and (iii) $\int_{\beta_{i}} d(\arg F(z))=0$. But $F_{0}(z)$ of [4] also uniquely maximizes $\Phi$ in the same class of functions. Hence $F_{0}(z)=G(z)$, and this completes the proof of Theorem 1 .

If we apply the corollary in the appendix, we find

COROLLARY 1. The set of mappings $\left\{F_{0}(z) \exp (2 \pi i / \lambda) k ; k=\right.$ $0,1, \cdots, \lambda-1\}$ represents all analytic $\lambda$-th roots of $F_{0}^{\lambda}(z)$. Also, the set of mappings $\left\{F_{1}(z) \exp (2 \pi i / \lambda) k ; k=0,1, \cdots, \lambda-1\right\}$ represents all analytic $\lambda$-th roots of $F_{1}^{\lambda}(z)$.

COROLLARY 2. The mappings $F_{0}^{\lambda}(z)$ and $F_{1}^{\lambda}(z)$ are respectively weakly $\lambda$-valent radial and circular slit disk mappings of $\bar{W}$.

COROLlaRY 3. For positive integers $\lambda$ and $\mu$, the relations 


$$
F_{i}^{\lambda \mu}(z)=\left(F_{i}^{\lambda}(z)\right)^{\mu}=\left(F_{i}^{\mu}(z)\right)^{\lambda}=\left(F_{i}(z)\right)^{\lambda \mu}
$$

hold for $i=0,1$.

Similar results may be obtained for $\lambda$-th principal analytic functions defined on $\bar{V}$, a bordered Riemann surface with two border components $\gamma$ and $\delta$. Again the construction of such functions is suggested by a known construction in the univalent case [3]. One starts with singularity functions $s_{\gamma}^{\lambda}(z)$ and $s_{\delta}^{\lambda}(z)$ defined near $\gamma$ and $\delta$ of the approximating bordered $\bar{V}_{n}$, and takes as further singularity functions $s_{\beta_{i}}^{\lambda}(z)$, the functions $s_{\beta_{i}}(z)$ of [3]. Here, $s_{\gamma}^{\lambda}(z)$ is constant on $\gamma$ with flux $2 \pi \lambda$, while $s_{\delta}^{\lambda}(z)$ is constant on $\delta$ with flux $-2 \pi \lambda$. An application of the linear operator method [5] to each $\bar{V}_{n}$ establishes the existence of the normal families $\left\{F_{0 n}^{\lambda}(z)\right\}$ and $\left\{F_{1 n}^{\lambda}(z)\right\}$, all subject to the condition $F_{i n}^{\lambda}(\zeta)=1$. Principal $\lambda$-th analytic functions $F_{0}^{\lambda}(z)$ and $F_{1}^{\lambda}(z)$ now result upon taking limits on $n$. We state the following characterization of these functions in terms of the principal functions $F_{0}(z)$ and $F_{1}(z)$ of [3].

THeorem 2. The mapping $\left.F_{0}^{\lambda}(z)\left(F_{1}^{\lambda} z\right)\right)$ is the $\lambda$-th power of the univalent principal mapping $F_{0}(z)\left(F_{1}(z)\right)$ of [3].

Appendix. We state, without proof, a well known characterization of those analytic functions which, on an open planar Riemann surface $W$, have analytic $\lambda$-th roots. Since such a surface may be conformally embedded in the complex plane, standard techniques of complex analysis [1] may be employed.

THEOREM. Let $f(z)$ be analytic and never zero on the open planar Riemann surface $W$ and let $\lambda$ be a positive integer. Then $f(z)$ has an analytic $\lambda$-th root in $W$ if and only if, for each cycle $\sigma \subset W, \lambda$ divides the integer $(1 / 2 \pi) \int_{\sigma} d(\arg f(z))$.

COROLlaRY. Let $f(z)$ be an analytic function which is never $z$ sro in $W$. If, for each cycle $\sigma \subset W, \lambda$ divides the integer $(1 / 2 \pi) \int_{\sigma} d(\arg f(z))$, then $f(z)$ has exactly $\lambda$ analytic $\lambda$-th roots in $W$.

\section{BIBLIOGRAPHY}

1. L. Ahlfors, Complex analysis, McGraw-Hill Book Company, Inc., 1952.

2. L. Ahlfors and L. Sario, Riemann surfaces, Princeton University Press, 1960.

3. P. Nickel, On extremal properties for annular radial and circular slit mappings of bordered Riemann surfaces, Pacific J. Math. 11, 1487-1503. 
4. L. Sario, Strong and weak boundary components, J. Analyse Math. 5, (1956-57), 389-398.

5. - A linear operator method on arbitrary Riemann surfaces, Trans. Amer. Math. Soc. 72 (1952), 281-295.

Received October 25, 1965, and in revised form January 17, 1966.

North Carolina State University 


\section{PACIFIC JOURNAL OF MATHEMATICS}

\section{EDITORS}

\author{
H. SAMELSON \\ Stanford University \\ Stanford, California \\ J. P. JANS \\ University of Washington \\ Seattle, Washington 98105
}

\section{J. DugundJI}

University of Southern California Los Angeles, California 90007

RICHARD ARENS

University of California

Los Angeles, California 90024

\section{ASSOCIATE EDITORS}
E. F. BECKENBACH
B. H. NeumanN
F. WOLF
K. YOSIDA

\section{SUPPORTING INSTITUTIONS}

\author{
UNIVERSITY OF BRITISH COLUMBIA \\ CALIFORNIA INSTITUTE OF TECHNOLOGY \\ UNIVERSITY OF CALIFORNIA \\ MONTANA STATE UNIVERSITY \\ UNIVERSITY OF NEVADA \\ NEW MEXICO STATE UNIVERSITY \\ OREGON STATE UNIVERSITY \\ UNIVERSITY OF OREGON \\ OSAKA UNIVERSITY \\ UNIVERSITY OF SOUTHERN CALIFORNIA
}

\author{
STANFORD UNIVERSITY \\ UNIVERSITY OF TOKYO \\ UNIVERSITY OF UTAH \\ WASHINGTON STATE UNIVERSITY \\ UNIVERSITY OF WASHINGTON \\ AMERICAN MATHEMATICAL SOCIETY \\ CHEVRON RESEARCH CORPORATION \\ TRW SYSTEMS \\ NAVAL ORDNANCE TEST STATION
}

Mathematical papers intended for publication in the Pacific Journal of Mathematics should be typewritten (double spaced). The first paragraph or two must be capable of being used separately as a synopsis of the entire paper. It should not contain references to the bibliography. Manuscripts may be sent to any one of the four editors. All other communications to the editors should be addressed to the managing editor, Richard Arens at the University of California, Los Angeles, California 90024 .

50 reprints per author of each article are furnished free of charge; additional copies may be obtained at cost in multiples of 50 .

The Pacific Journal of Mathematics is published monthly. Effective with Volume 16 the price per volume (3 numbers) is $\$ 8.00$; single issues, $\$ 3.00$. Special price for current issues to individual faculty members of supporting institutions and to individual members of the American Mathematical Society: $\$ 4.00$ per volume; single issues $\$ 1.50$. Back numbers are available.

Subscriptions, orders for back numbers, and changes of address should be sent to Pacific Journal of Mathematics, 103 Highland Boulevard, Berkeley 8, California.

Printed at Kokusai Bunken Insatsusha (International Academic Printing Co., Ltd.), No. 6, 2-chome, Fujimi-cho, Chiyoda-ku, Tokyo, Japan.

PUBLISHED BY PACIFIC JOURNAL OF MATHEMATICS, A NON-PROFIT CORPORATION

The Supporting Institutions listed above contribute to the cost of publication of this Journal, but they are not owners or publishers and have no responsibility for its content or policies. 


\section{Pacific Journal of Mathematics \\ Vol. 20, No. $2 \quad$ October, 1967}

Edward Dewey Davis, Ideals of the principal class, $R$-sequences and a certain monoidal transformation ............................. 197

Richard Mansfield Dudley, Sub-stationary processes ................ 207

Newton Seymour Hawley and M. Schiffer, Riemann surfaces which are doubles of plane domains ......................... 217

Barry E. Johnson, Continuity of transformations which leave invariant certain translation invariant subspaces ................... 223

John Eldon Mack and Donald Glen Johnson, The Dedekind completion of

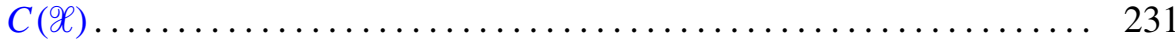

K. K. Mathur and R. B. Saxena, On the convergence of quasi-Hermite-Fejér interpolation................................... 245

H. D. Miller, Generalization of a theorem of Marcinkiewicz............ 261

Joseph Baruch Muskat, Reciprocity and Jacobi sums ............... 275

Stelios A. Negrepontis, On a theorem by Hoffman and Ramsay .......... 281

Paul Adrian Nickel, A note on principal functions and multiply-valent canonical mappings .............................. 283

Robert Charles Thompson, On a class of matrix equations ............. 289

David Morris Topping, Asymptoticity and semimodularity in projection lattices ........................................ 317

James Ramsey Webb, A Hellinger integral representation for bounded linear functionals

Joel John Westman, Locally trivial $C^{r}$ groupoids and their representations...

Hung-Hsi Wu, Holonomy groups of indefinite metrics 